dangers of infection, they would at once apply to a physician should the slightest symptoms appear.

To return to the relation of the physician to the State. That physicians are able to do the most for the prevention of venereal diseases is easily demonstrated, but only when the Government arrives at the conviction that the profession should be consulted and their advice accepted. In the first place the sale of all proprietary medicines for venereal diseases should be prohibited and quack advertisers suppressed, while druggists should not be allowed to prescribe. The law in this respect should be strictly enforced and the part of the physician will be a much easier matter. He should not deliver a prescription to a patient calling for a specific remedy. He (the doctor) should either furnish the remedy himself, or, if it is dispensed by the druggist, it should pass through the hands of the physician.

To substantiate what $I$ have just said, take, for example, a case of acute gonorrhea. The patient receives his prescription and possibly returns a second time, to disappear for ever. He does not consult another physician, but has his prescription filled and refilled until the alarming symptoms, copious dis. charge and intense burning subside. The chronic gonorrhea ensuing does not cause him as much distress, and possibly a morning drop only remains. He deems himself cured and seeks new cohabitation, and naturally not where he contracted his first infection, and so-transmits the disease to one or more. Besides, he may have a good friend in like condition whom he is happy to advise, and the original prescription is refilled indefinitely with a similar result each time. In all cases, where it is possible, the patient should be treated in the doctor's office. In the first place, the application will be properly made, and secondly, he will have the moral support and good counsel of the doctor to deter him for at least the next twentyfour hours from indulging in liquors or cohabitation. The danger of complications being frequently emphasized and impressed upon him, he becomes at length more and more conscious of the fact that he may be a source of danger to others and learn to weigh his responsibilities. And finally, he is not discharged until cured. The same must be said of female patients and the effect of severe medical control will be equally observed. But how with patients whom circumstances compel to change places, for instance, traveling men? They have the opportunity to consult a physician wherever they go, and he can do the same. If a patient lacks funds to pay the usual fees, he will still find a liberal physician who will attend to his case. A patient without any means should apply to a doctor for a certificate directing him to a dispensary or hospital. Of these institutions there should be a sufficient number according to population, and supported by the Government with sufficient liberality to afford the patient the best of care. An ample staff of experienced physicians should be elected by the profession-and not through political influence-to attend these institutions, and should receive suitable remuneration. Thus, at least, in one branch of medicine there would be avoided the abuse of the free dispensary by persons perfectly competent to pay, and from these hospitals and dispensaries the necessary clinical material for instruction could be secured.

Impracticable as some of the foregoing suggestions may appear, it is still true that no material advantage is obtained without effort, and considering the enormous benefit to humanity, it can not surely be purchased at too great a price.

103 State Street.

\section{SOME FORMS OF GANGRENE AND THEIR TREATMENT.}

Read before the Mississippi Valley Medical Association, Nashville, Tenn, Oct. 11-16, 1898 .

BY J. S. NOWLIN, M.D. SHELBYYILLE, TENN.

Gangrene means death of a part, and by common consent is technically applied to the soft tissues, including the fluids. The blood is always involved in this disease, and there can be no death of a part while it is performing its function. With this thought kept before us, that there is no local death where the blood is performing its function, we may inquire into the conditions which result in gangrene.

External violence may crush the tissues, to the ex. tent that circulation is theroby arrested. The channels for the blood-current being destroyed, it is impossible for it to yield its life-giving force, and the part must necessarily die. So long as the arteries and their capillary system are at all intact, life may con. tinue and the injury be repaired. The same may be produced by the extremes of heat and cold, burning and freezing; the tissues being destroyed, the blood can not circulate. These we class as traumatic causes of gangrene. We also have internal conditions result. ing in gangrene, e.g., the neuropathic form, in which death of a part results from sudden withdrawal of the nutritive forces.

Though I am not sustained by the weight of author. ity, I believe we have a trophic nerve which controls the nutritive forces, and directs that energy of the circulating medium which feeds the system and gives life. Some have looked upon this as inherent in the common function of the vasomotor system. I believe there is a receptive vital force inherent in the cell which enables it to receive and appropriate nutritive energies of the blood as it passes by, and if this is wanting in a given tissue, the blood, though normal, can not impart its life, and death at this point is the result. I saw decubitus appear on the nates of a fee. ble old lady aged 70 years, within twenty-four hours after she suffered a fracture of the neck of the fermur. It also appears quickly after injuries to the spinal cord. It is patent to every close observer that when a part has been injured as, for example, an incised wound, the blood is quickly rushing to its relief, and all the capillaries in close proximity are immediately surcharged. This is not simply that continuity is wanting, and the vessels leaking, but it is an intelligent determination of the life-giving fiuid for purposes of repair. This is the first step of all the steps in the process of that series of conditions which we call inflammation, and, if recognized and understood by the surgeon will, as a rule, be the only step.

Simple endarteritis is sometimes the cause of gan. grene. In this condition it is not because the lumen has been obliterated, but because the intima is not in receptive status and the blood can not impart its nutrition. Vitality of the tissues is thus rapidly im. paired and lost, the sequence being that the blood is retained in the artery to an extent beyond its capacity. The inflamed artery is thus surcharged with blood, and a stasis results which ends in coagulation, ob- 
structing the lumen to an extent commensurate with the inflammation. Here the gradual loss of life precedes the actual infarction of the vessel. In support of this I quote from Warren, who says: "Individuals affected with diabetes or scurvy, are peculiarly liable to gangrenous processes."

The so-called marasmic thrombi are usually due to a slowing of the current, and a coincident defect of the walls of the blood-vessels, thus favoring coagulation. The same thought is in Weigert's theory of coagulation neurosis, in which he says that the cells of the tissue become altered to granular or hyaline masses and lose their nuclei. The obliterative endarteritis, with new tissue formation, has impressed me as a pathologic condition which has not grown up suddenly, but requires time.

Sepsis is doubtless the more frequent cause of gangrene in the extremities, in cases of continued fever, or any protracted trouble in which blood is spanemic and the nervous system depressed.

Senile gangrene is the result of diseased arterial walls being either atheromatous or deficient in nervesupplies, possibly both. It will be observed that sepsis may produce thrombosis, and either this or embolus may obstruct the arterial canal at the extremities. The difference then from senile gangrene is in that form producing cessation of nutrition because of non. nutritious capillaries, while the septic form is from thrombosis. The time for operative treatment in sen. ile gangrene is somewhat at the discretion of the surgeon, but in the septic or obstructive form, it must be done instantly.

In the line of treatment the time for operative interference which will be safe to life, and for determining the point of amputation which will least mutilate the body, are all important. If traumatic in any of the varieties, we know that it is local, and so long as it so continues, there is no danger of constitutional effects that will be destructive to life. The operator should be familiar with the earliest indications of sepsis, as also that traumatic endarteritis, known as fulminating, which now and then destroys the patient so quickly. The artery is lacerated or in some way wounded, and the rapid obliteration may be caused by local shock to the trophic nerve, or the intima may become at once inflamed, with like result. When this state exists, amputation should be done at once at a point above bifurcation. On the other hand, if the above does not exist, the surgeon may select his own time for operation.

In the senile form, the disease is caused by sclerotic conditions of the arterial wall, whereby the expansive and contractive function is destroyed, and the nutritive receptivity lost; the part dies for want of nutrition, though the blood is normal. Twenty-five years ago I was afraid to cut a sclerotic artery, but now I am sure the best practice is to amputate.

In the beginning of this paper attention was directed to the blood as the leading factor to be looked after in pathology and treatment. If this is kept in view, the question of demarcation will no longer be looked upon with so much anxiety. There are four principal causes: 1 , the septic, in which the blood is diseased; 2 , the senile or atheromatous condition; 3 , trauma; 4 , inflammation of the internal coat of the artery. If septic, we have thrombi and coagulated blood up to the first principal bifurcation of the artery. The symptoms being pain first, then coldness and ease, are all that ought to be asked, and if these obtain, amputate high up at once. If senile or sclerotic, a little time may be given, but the knife must come, and that above the ankle if not higher. If traumatic, the watchful intelligent eye and tactus eruditus must be on the outlook for sepsis and hold the knife in constant readiness to assist nature at the first intimation. If simple endarteritis, or endarteritis with tissue formation, then again amputate at the first evidence of fading vitality.

\section{HYDATID CYST OF THE LIVER.}

LAPARO-HEPATOTOMY AND RECOVERY.

Read before the Indiana State Medical Society, May 28 and 29, 1896. BY LEON J. WILLIEN, M.D. TERRE HAUTE, IND.

The history of echinococci of the liver, or hydatids, is of no recent date, because Hippocrates describes certain kinds of fluid he found in the liver, which description, from its preciseness, leads us to believe that the malady existed then. Both Galen and Aretæus were not ignorant of its existence, yet no records could be found giving an accurate description of these cysts until more attention was given to the study of anatomy, in the sixteenth and seventeenth centuries, when Plater, Vega and Riverius gave a more careful and minute description of the disease. To Breemer is due the credit of having first given an accurate description of the echinococci, in 1821. From that year on, with the progress and rapid advancement of medical science, more was learned of this hepatic parasite, and the microscope reveals its existence.

I am not desirous of dragging this paper into a lengthy description of the disease, but simply ask attention to the following case, which, on account of its rarity and the result, was one of unusual interest to my fellow-colleagues and myself. To make a diagnosis of a hydatid tumor by a simple physical exam. ination is difficult, unless we have recourse to the microscope, otherwise there there would be a probability of its being not a certainty. Even the closest clinical observation, kept up for days, will often keep the physician at sea as to the certainty of the diagnosis; therefore we must always accept cum grano salis the positive existence of a hydatid tumor of the liver unless a microscopic examination of the contents is made. How do we know for certain whether the liquid withdrawn from the pleural cavity is serum, or whether a hydatid sac has broken into it, without that examination? The symptoms would be those of a sudden rupture. They would quickly dispel the idea or the surmise of an effusion resulting from pleuritis. The diagnosis of such a case has been made most frequently at the postmortem.

Case 1.-Elias W., aged 50 years, single, coal miner by occupation, residing at Fontanet, Vigo Co., Ind., small in size, thin in flesh, light complexion, and of a delicate constitution. $\mathrm{He}$ claimed to be of regular habits; his physiognomy is charac. teristic of an eccentric individual, a religious crank, and a poet. Could find out very little concerning his history. For some months previous to his consulting a physician he had pain in the region of the liver, with indigestion, constipation, and a sensation of weight in the hepatic region. The pain came on gradually and ceased suddenly. He was admitted to St. Anthony's Hospital June 26,1895, and placed in the clinical ward, then in the charge of Dr. Walker Schell.

The history of the case was rather obscure, he saying that he had pains in the region of the liver since September, 1894. After observation for several days the Doctor discovered an unusual increase of the anterior lobe of the liver. The larger diameter of the tumor was felt just over the juncture of the nipple and the parasternal lines. Its face was irregular, soft, and almost fluctuating to the touch; the area of the stomach 